University of Nebraska - Lincoln

DigitalCommons@University of Nebraska - Lincoln

Nutrition and Health Sciences -- Faculty

Publications

Nutrition and Health Sciences, Department of

2005

\title{
Omega-3 Fatty Acid Consumption and Food Sources Differ among Elderly Men Living in Coastal and Internal Regions of Saudi Arabia
}

Khalid AL-Numair

King Saud University, Riyadh, Saudi Arabia, Kalnumair@hotmail.com

Nancy M. Lewis

University of Nebraska--Lincoln, nlewis2@unl.edu

Sharon Evans

University of Nebraska - Lincoln, sevans1@unl.edu

Follow this and additional works at: https://digitalcommons.unl.edu/nutritionfacpub

Part of the Dietetics and Clinical Nutrition Commons

AL-Numair, Khalid; Lewis, Nancy M.; and Evans, Sharon, "Omega-3 Fatty Acid Consumption and Food Sources Differ among Elderly Men Living in Coastal and Internal Regions of Saudi Arabia" (2005).

Nutrition and Health Sciences -- Faculty Publications. 10.

https://digitalcommons.unl.edu/nutritionfacpub/10

This Article is brought to you for free and open access by the Nutrition and Health Sciences, Department of at DigitalCommons@University of Nebraska - Lincoln. It has been accepted for inclusion in Nutrition and Health Sciences -- Faculty Publications by an authorized administrator of DigitalCommons@University of Nebraska - Lincoln. 
Pakistan Journal of Nutrition 4 (2): 106-111, 2005

ISSN 1680-5194

(C) Asian Network for Scientific Information, 2005

\title{
Omega-3 Fatty Acid Consumption and Food Sources Differ among Elderly Men Living in Coastal and Internal Regions of Saudi Arabia
}

\author{
Khalid AL-Numair ${ }^{1}$, Nancy M. Lewis ${ }^{2}$ and Sharon Evans ${ }^{3}$ \\ 'Department of Food Sciences and Nutrition, King Saud University, P.O. Box 2460 Riyadh, Saudi Arabia \\ ${ }^{2}$ Departmen of Nutrition and Health Sciences, ${ }^{3}$ Department of Educational Psychology, \\ College of Education and Human Sciences, \\ University of Nebraska, Lincoln, Nebraska, 68583, USA \\ E-mail: Kalnumair@hotmail.com
}

\begin{abstract}
Research suggests that omega-3 fatty acids ( $n-3$ FAs) play an important role in reducing the risk of heart diseases. The objectives of the current study were to investigate the consumption and the food sources of n-3 FAs in two samples of elderly men living in different geographic locations (coastal and internal regions) of Saudi Arabia. Sixty men from each of the coastal and the internal regions provided a 24-hour recall and 2-day food record and a food frequency questionnaire. Food Processor Plus computer program software (ESHA Version 0.7; Salem, Oregon, 2002) was used to analyze the dietary intake. The intake of total n-3 FAs, alpha-lenolenic acid (ALA), eicosapentaenoic acid (EPA) and docosahexaenoic acid (DHA) were higher among the coastal region residents than among the internal residents $(P<0.05)$. English walnuts, salmon, canola oil, malabar cavalla and king mackerel were the top five foods contributing to the n-3 FA intakes in the coastal region. The top five foods contributing to the n-3 FA intakes in the internal region were English walnuts, lamb, whole milk, baked beans and chicken. The food contributing the most to the intake of $\mathrm{n}-3 \mathrm{FAs}$ in both regions were English walnuts. In conclusion, the food consumption pattern for food providing n-3 FAs differs by location and coastal residents consume more than twice as much n-3 FAs as internal residents. Nutrition education intervention among internal residents is needed for increasing the consumption of $n-3$ FAs.
\end{abstract}

Key words: Coastal region, omega-3 fatty acids, Saudi Arabia, sea food

\section{Introduction}

Saudi Arabia (SA) lies at the furthermost part of southwestern Asia. It occupies about $80 \%$ of the Arabian Peninsula, with a total area of over 2,250,000 square kilometers (Statistical Yearbook, 1999), and is at the crossroads of three continents-Europe, Asia and Africa. Based on its climate, Saudi Arabia can be divided into two main regions, coastal and internal. The coastal regions lie close to the coasts of Arabian Gulf on the east (known as Al-Sharqia) and to the Red Sea on the west (known as Al-Garbia). All other regions can be categorized as internal or interior regions (Saudi Arabian Information Resource, 2002).

Diet has long been considered to play a critical role in human health. Geographic location has an impact on the consumption of seafood and n-3 FAs. (Bulliyya et al., 1990; Torres et al., 2000).

Interest in n-3 FAs has increased over the past two decades because of potential health benefits. N-3 FAs are polyunsaturated fatty acids (Horrocks and Yeo, 1999). There are three n-3 FA that are ingested in foods, alpha lenolenic (ALA) from plant sources, docosahexaenoic acid (DHA), eicosapentaenoic acid (EPA) provided from fish. Human cells can elongate and desaturate ALA to produce EPA and DHA (Gerster, 1998;
Holub, 2002). For this reason ALA is considered an essential fatty acid, i.e. being essential to human health but cannot be manufactured by the body and thus must be obtained from food. Most fish have a high content of n-3 FAs and geographic location has an impact on the consumption of seafood. Geographic location therefore affects intakes of n-3 FA (Bulliyya et al., 1990).

Epidemiological and clinical trials suggest that $n-3$ FAs have a significant role in the prevention of coronary heart disease (CHD). In the 1970s, epidemiological studies related the low incidence of ischemic heart disease in Greenland Eskimos with their traditional diet, which consists almost exclusively of seafood (Dyerberg et al., 1978; Bang et al., 1980). In a prospective population study an inverse association between fish consumption and the 30-year risk of death from CHD was also reported (Daviglus et al., 1997). An inverse association between fish intake and n-3 FAs intake and CHD death was also reported in the Nurses' Health Study (Hu et al., 2002).

The largest prospective study to test the efficacy of $n-3$ FAs on the prevention of CHD is the GISSI-Prevention Study. After 3.5 years of follow-up there was a $15 \%$ reduction in the primary end point for a combination of death, nonfatal myocardial infarction, and nonfatal stroke 


\section{AL-Numair et al.: Omega-3 Fatty Acid Consumption and Food Sources}

in those receiving $0.85 \mathrm{~g}$ of $\mathrm{n}-3$ FAs per day as $\mathrm{DHA}+\mathrm{EPA}$ (GISSI, 1999).

The importance of ALA was identified in the cohort study by Dolecek (1992), who reported a significant inverse association for the intake of alpha-linolenic acid (ALA) and mortality rates from $\mathrm{CHD}$ in 6250 men. Also, the importance of ALA was reported in the Nurses Health Study by $\mathrm{Hu}$ et al. (1999). They reported that a higher intake of food containing ALA was associated with a lower relative risk of fatal $\mathrm{CHD}$.

In a second study by $\mathrm{Hu}$ et al. (1998) women who ate more than $142 \mathrm{~g}$ of nuts per week had a significantly lower risk of total CHD than women who never ate nuts or ate less than $28 \mathrm{~g}$ per month. In a recent cohort study, an association of higher serum concentration of ALA with a lower risk of fatal ischemic heart disease in older adults was documented (Lemaitre et al., 2003).

Evidence also indicates that $n-3$ FAs reduce inflammation (James et al., 2000; Gil, 2002; Simopoulos, 2002) and they influence ventricular fibrillation (Charnock, 1999), vasodilatation, and thrombosis (Horrocks and Yeo, 1999). There are also reports that n-3 FAs reduce plasma triacylglycerol levels (Harris, 1996) and lower blood pressure (Mori et al., 1999; Howe, 1997).

Seafood such as king mackerel, salmon, tuna, sardines and shellfish are the main dietary source of EPA and DHA (Nettleton, 1991; Kotab et al., 1991). The precursor of these long chain n-3 FA, ALA, is found in canola oil, walnut oil, flaxseed oil, soy oil, legumes such as baked beans and navy beans (Nettleton, 1991) and most green leafy vegetables such as spinach, broccoli, watercress and cabbage (Duo and Sinclair, 2001). Fruits such as avocados, cherries, and strawberries also provide ALA (Harper and Jacobson, 2001).

Dietary recommendations for n-3 FAs have not been established in Saudi Arabia; however, recommendations have been established recently in the United States [Dietary Reference Intakes (DRI), 2002]. Public health agencies in Saudi Arabia can utilize information on the consumption and sources of n-3 FA among the population. Moreover information from this study will assist in the design and implementation of educational interventions for people who have low n-3 FA intakes or those who are at potential risk of CHD.

Therefore the purpose of this study was to determine the consumption of $n-3$ FAs and the food sources of n-3 FAs in two samples of elderly men living in diverse geographical locations, i.e. coastal versus internal, in Saudi Arabia.

\section{Materials and Methods}

Selection of subjects: Elderly men from coastal and internal regions of Saudi Arabia were recruited. The coastal region is close to the Arabian Gulf and the internal region represents the Central Part of Saudi
Arabia. Since there was no available data on the intake of n-3 FA in Saudi Arabia, which could have help determine the sample size. Therefore a tenth percent $(0.001)$ were selected from the total number of men aged 60 and over years in the coastal region $(n=60)$ then another sixty men aged 60 and over years were selected from internal region.

The coastal cities sampled were Dhahran, Dammam, and Qatif, and internal cities were Buraida, Unaiza, and Bukerah. Sixty male subjects aged $\geq 60$ years, twenty from each city, were randomly selected from the telephone directory of each geographical region. Subjects age was identified by calling the randomly selected number and asked if there was a man aged sixty or over willing to participate in the study. If there was not a man aged sixty and over, another number was randomly selected until the sample number were recruited. All subjects contacted volunteered to participate in the study. The procedures for the study were approved by the University Institutional Review Board.

Dietary intake and food frequency questionnaire: During summer of 2001 each volunteer was interviewed and provided demographic information and food intake information. Demographic information collected included age, education level, marital status, and monthly income. The demographic information form was filled by the researcher during the interview. Two nutritionists reviewed the demographic form for content validity.

During the interview a nutritionist provided instructions on estimating serving sizes using plastic food models. One 24-hour food recall was completed during the interview and volunteers were instructed to complete a two-day food record so that three non-consecutive days of intake were obtained. Food intake for the two food records was collected by telephone. The Food Processor Plus computer program software (ESHA Version 0.7; Salem, Oregon, 2002) was used to analyze the food record and to calculate the mean daily omega-3 fatty acid intake.

During summer of 2002 each volunteer completed a food frequency questionnaire. The food frequency questionnaire was developed by listing the following food categories which contain n-3 FAs : seafood, vegetables, fruits, bread and cereal products, meat, eggs, legumes, nuts and seeds, and dairy by using the Food Processor Plus computer program software (ESHA Version 0.7; Salem, Oregon, 2002) and Kotab et al., 1991. Foods that are commonly consumed by individuals in both regions were analyzed by the computer software package, Food Processor Plus, 2002 for their amount of $n-3$ FAs. A medium serving size was determined using the United States Department of Agricultures' Food Guide Pyramid, 2000. 


\section{AL-Numair et al.: Omega-3 Fatty Acid Consumption and Food Sources}

Table 1: Demographic Characteristics of Elderly Men Living in Two Regions of Saudi Arabia

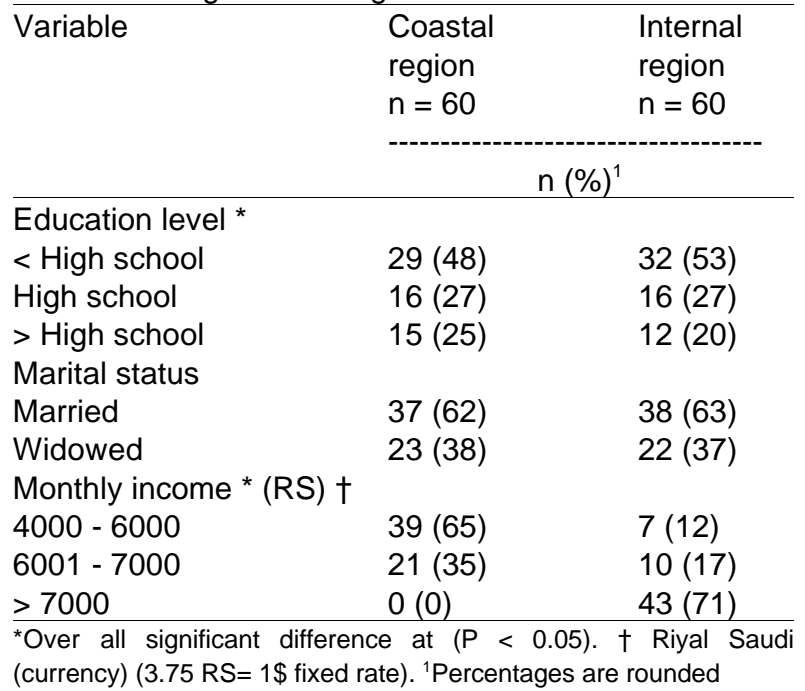

Table 2: Omega-3 Fatty Acid Intakes of Elderly Men \begin{tabular}{lll}
\multicolumn{2}{c}{ Living in Two Regions of Saudi Arabia } \\
\hline Variable $^{1}$ & $\begin{array}{l}\text { Coastal } \\
\text { region }\end{array}$ & Internal \\
& $n=60$ & $n=60$ \\
& & region \\
& &
\end{tabular}

\begin{tabular}{lll} 
& \multicolumn{2}{c}{ Mean \pm SD } \\
\hline Total n-3 FAs (g/day) & $2.18 \pm 0.58^{\mathrm{a}}$ & $0.79 \pm 0.13^{\mathrm{b}}$ \\
ALA (g/day) & $0.90 \pm 0.12^{\mathrm{a}}$ & $0.71 \pm 0.10^{\mathrm{b}}$ \\
EPA (g/day) & $0.38 \pm 0.16^{\mathrm{a}}$ & $0.03 \pm 0.02^{\mathrm{b}}$ \\
DHA (g/day) & $0.88 \pm 0.30^{\mathrm{a}}$ & $0.06 \pm 0.03^{\mathrm{b}}$
\end{tabular}

ab values with different letters in the same row are significantly different at $p<0.05$.

${ }^{1} \mathrm{n}-3$ Fas $=$ Omega-3 fatty acids, ALA = alpha-linolenic acid, EPA = eicosapentaenoic acid, and DHA = docosahexaenoic acid

Consumption of food was recorded for each category based on number of servings per week of specific foods consumed. Two nutritionists reviewed the food frequency questionnaire for content validity. Volunteers were asked to indicate how often they had consumed each food item during the current survey week. We used a time period of one week to accommodate the age of our volunteers (Beaton et al., 1997).

Data analysis: Data for the coastal and internal residents were compared using Statistical Package for the Social Sciences computer program software (SPSS for Windows, version 11.0, SPSS, Inc, 2002). Descriptive statistics were used to display data in frequency, percentages and means. Chi-square was used to compare demographic information such as education level, marital statues and monthly income between the two samples in both regions. Student's t-test was used to compare n-3 FA intakes between the two samples. The $p$ - value used to accept a significant result was $p<$ 0.05 .
Results

Demographic: The mean age of coastal residents (mean $\pm \mathrm{SD})(66.5 \pm 2.23$ years) was older than that of internal residents (63.8 \pm 0.97 years) $(P<0.05)$. Education and income levels were also different for residents of the two regions $(P<0.05)$ (Table 1$)$. Residents of the internal region had less education and higher incomes.

Omega-3 fatty acids intake: Table 2 shows a comparison of n-3 FA intakes among men in the two regions. Coastal residents consumed more than twice as much n-3 FAs as internal residents $(P<0.05)$. Consumption of ALA, EPA and DHA were also significantly higher in coastal residents. Mean n-3 FAs consumption was more than the recommended intake for residents of the coastal region $(2.18 \pm 0.58)$ (g/day), but was $49 \%$ of the DRI recommendation for residents of the internal region (DRI, 2002).

Food frequency questionnaire: Table 3 shows that English walnuts, salmon, canola oil, malabar cavalla and king mackerel were the top five foods contributing to the intakes of n-3 FA in the coastal region. Seafood was a major contributor to the intakes of n-3 FA in the coastal region as three-fourths of the residents in this region consumed 5-7 seafood servings/week.

The type of fish contributing the most to the n-3 FA intakes in the coastal region was salmon. As a source of n-3 FAs all fish in the seafood category provided 0.40 g/day. English walnuts, zucchini squash, cherries, baked beans and canola oil were the plants contributing the most to the intakes of $n-3 F A$ in the coastal region and they provided $0.36 \mathrm{~g} /$ day. Lamb, chicken, whole milk and cheddar cheese were other foods within the top fourteen foods contributing to the intake of $n-3$ FAs in the coastal region. These foods provided $0.13 \mathrm{~g} \mathrm{n}-3 \mathrm{FA} /$ day.

Table 4 shows that residents in the internal region indicate no consumption of seafood. The top five foods contributing to the n-3 FAs intake in the internal region were English walnuts, lamb, whole milk, baked beans and chicken. After English walnuts, the plant foods contributing the most to the intake of $n-3$ FAs in the internal region were zucchini squash, potato, spinach, cherries, cantaloupe, apple and baked beans. Together plant food among the top 14 foods contributing to the intake of $n-3$ FAs in the internal region provided 0.28 g/day. Lamb, chicken, egg, whole milk, cheddar cheese and butter were other foods included in the top fourteen foods contributing to the intakes of $n-3 F A$ in the internal region; together these provided $0.26 \mathrm{~g} /$ day.

\section{Discussion}

Results of this study of residents in coastal and internal regions of Saudi Arabia indicate that the consumption patterns for food providing n-3 FA differ by region of the country. N-3 FA intakes was higher in the coastal region 


\section{AL-Numair et al.: Omega-3 Fatty Acid Consumption and Food Sources}

Table 3: Foods Contributing to the Intakes of N-3 Fatty Acids in Diets of Elderly Men Living in Coastal Regions of Saudi Arabia

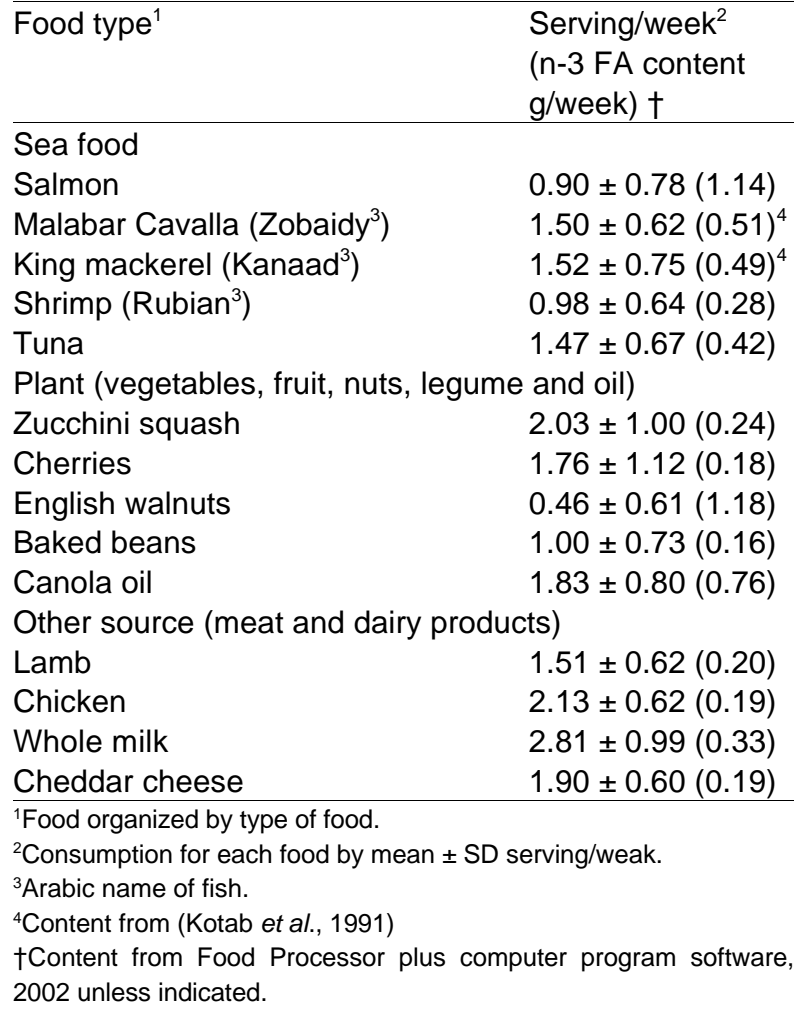

Table 4: Foods Contributing to the Intake of N-3 Fatty Acids in Diets of Elderly Men Living in Internal Regions of Saudi Arabia

\begin{tabular}{lc}
\hline Food type $^{1}$ & $\begin{array}{l}\text { Serving/week } \\
(\mathrm{n}-3 \mathrm{FA} \text { content } \\
\text { g/week) } \dagger\end{array}$ \\
\hline Plant (vegetables, fruit, nuts and legume) \\
Zucchini squash & $1.75 \pm 0.89(0.21)$ \\
Potato & $2.65 \pm 1.63(0.12)$ \\
Spinach & $2.33 \pm 1.56(0.12)$ \\
Cherries & $1.88 \pm 1.15(0.19)$ \\
Cantaloupe & $1.93 \pm 0.75(0.14)$ \\
Apple & $2.93 \pm 1.26(0.09)$ \\
English walnuts & $0.30 \pm 0.49(0.77)$ \\
Baked beans & $0.90 \pm 1.00(0.30)$ \\
Other source (meat and dairy products) \\
Lamb & $7.51 \pm 0.70(0.75)$ \\
Chicken & $3.31 \pm 1.45(0.29)$ \\
Egg & $2.91 \pm 0.88(0.12)$ \\
Whole milk & $2.98 \pm 1.14(0.35)$ \\
Cheddar cheese & $1.30 \pm 0.64(0.13)$ \\
Butter & $3.60 \pm 1.04(0.21)$ \\
\hline${ }^{1}$ Food organized by type of food. & \\
${ }^{2}$ Consumption for each food by mean \pm SD serving/weak. \\
$\dagger$ Content from Food Processor Plus computer program software, \\
2002.
\end{tabular}

and seafood was not consumed in the internal region. Geographic location has an impact on the consumption of seafood and n-3 FAs. This was also identified by Bulliyya et al. (1990) who reported that consumption of fish is not part of the food pattern of inland populations when compared with coastal populations in India. Torres et al. (2000) also provided evidence of the influence of geographic location on the n-3 FAs consumption. They reported higher intakes of n-3 FA among residents of a coastal village compared to a rural village on a Portuguese island.

In elderly Saudi Arabian men intakes of n-3 FAs, EPA, DHA, and ALA were higher among those living in the coastal region than among those in the internal region. Mean n-3 FA consumption was more than the recommended intake for the elderly men in the coastal region $(2.18 \pm 0.58 \mathrm{~g} /$ day $)$, but was less than optimum for the internal region $(0.79 \pm 0.13 \mathrm{~g} /$ day $)$ where the consumption was $49 \%$ of the dietary reference intake (DRI) recommendation (DRI, 2002).

There are no published research data available on the dietary intakes of n-3 FA in Saudi Arabia, however several studies have been conducted in other countries. The impact of geographic location was shown by Torres et al. (2000) in a Portuguese island comparing the dietary intake of two populations of men with age ranging from 25 to 65 years, and with different dietary habits (fishing village vs. rural village). Torres et al. (2000) reported a higher consumption of ALA, DHA and EPA in the fishing village. However, consumption of ALA $(0.49 \pm 0.30 \mathrm{~g} /$ day $)$, EPA $(0.22 \pm 0.36 \mathrm{~g} /$ day $)$, and DHA $(0.54 \pm 0.79 \mathrm{~g} / \mathrm{day})$ of men in the Portuguese fishing village was approximately one half the consumption by elderly men in the coastal region of Saudi Arabia. Consumption of ALA was low in the Portuguese rural village $(0.35 \pm 0.31 \mathrm{~g} /$ day $)$ and was approximately $50 \%$ of that consumed by elderly men in an internal region of Saudi Arabia ( $0.71 \pm 0.10 \mathrm{~g} /$ day). Consumption of EPA $(0.03 \pm 0.07 \mathrm{~g} /$ day $)$ and DHA $(0.05 \pm 0.07 \mathrm{~g} /$ day $)$ of male residents in the Portuguese rural village were similar to the consumption by elderly men in internal regions of Saudi Arabia.

Intakes of n-3 FA of 25 male and female volunteers with hypercholesterolemia living in the Midwestern United States (Lewis et al., 2000) were similar to the intakes by elderly Saudi Arabian men living in the internal region of the country $(0.62 \pm 0.07 \mathrm{~g} / \mathrm{day})$ and $(0.79 \pm 0.13 \mathrm{~g} / \mathrm{day})$ respectively.

Another survey of 30 low-income, pregnant women living in the Midwest (Lewis et al., 1995) also provides evidence for the impact of geographic location on $n-3$ FAs intake. In this group, ALA intake ( $0.98 \pm 0.30 \mathrm{~g} /$ day $)$ was the major form of $n-3$ FA consumed. Intakes of EPA $(0.02 \pm 0.06 \mathrm{~g} / \mathrm{day})$ and DHA $(0.04 \pm 0.08 \mathrm{~g} / \mathrm{day})$ contributed very little to total $n-3$ FA intakes in this 


\section{AL-Numair et al.: Omega-3 Fatty Acid Consumption and Food Sources}

population. This is consistent with consumption by elderly Saudi Arabian men living in the internal region of the country, where the intake of ALA $(0.71 \pm 0.10 \mathrm{~g} /$ day $)$ was the major form of $n-3$ FAs intake, and EPA (0.03 \pm $0.02 \mathrm{~g} /$ day $)$ and DHA (0.06 $\pm 0.03 \mathrm{~g} /$ day) contributed very little. In both populations $\geq 90 \%$ of the $n-3 F A$ intake was provided by ALA.

Evidence from these four studies in three different countries indicates that geographic location affects $n-3$ FA intakes. Residents living far from coastlines have low intakes of total n-3 FAs, EPA, and DHA.

In the current study residents in the internal region of Saudi Arabia consumed no seafood. These results were similar to those of Bulliyya et al., 1990 and Torres et al., 2000 who reported that fish is not part of the food pattern in populations living far away from the coast.

In the current study fish contributed to the n-3 FAs intake only in the coastal region. Three-fourths of the residents in the coastal region consumed 5-7 fish servings/week of fish considered to be a good source of n-3 FA such as Malabar cavalla (Zobaidy), King mackerel (Kanaad), tuna and salmon (Nettleton, 1991; Kotab et al., 1991). The American Heart Association recommends fish twice per week (Kris-Etherton et al., 2002). Geographic location may be one of the reasons for the absence of fish consumption in the internal region. As a result diets are lower in DHA and EPA in the internal region. Bulliyya et al., 1990 and Torres et al., 2000, also reported lower daily consumption of EPA and DHA for those who live far way from the coastline.

English walnuts, zucchini squash, potato, cherries, cantaloupe, apple, and baked beans were plants food among top 14 foods contributed to the n-3 FA intakes in the internal regions. English walnuts, zucchini squash, cherries, baked beans and canola oil were the plants food within the top fourteen foods contributing to the intake of $n-3$ FA in the coastal region. The food contributing the most to the intakes of n-3 FA in both regions was English walnuts. English walnuts contain $2.57 \mathrm{~g}$ of $\mathrm{n}-3$ FA as ALA per one ounce serving (Food Processor Plus computer program software. ESHA Version 0.7, Salem, Oregon, 2002).

Plant sources contain ALA (Gerster, 1998) therefore the absence of seafood and contribution of plant source may explain why ALA was the major contributor to the $n-3$ FAs intake in internal region. ALA intake has been associated with a reduced risk of CHD in many studies (Dolecek, 1992; Prineas et al., 1993; Hu et al., 1999; Lemaitre et al., 2003).

Increasing the consumption of plants considered to be a good source of $n-3$ FAs such as English walnuts and canola oil (Nettleton, 1991) will increase the ALA intake and may be protective against CHD (Dolecek, 1992; Prinease, 1993; Hu FB et al., 1999; Lemaitre et al., 2003) this could be important for residents of the especially internal region because fish is not consumed. ALA is elongated and desaturated by the body to produce EPA and DHA (Gerster, 1998 and Holub, 2002). Therefore people who do not eat fish can increase their dietary n-3 FAs from plant sources. The animal foods chicken, lamb, cheddar cheese, and whole milk were other dietary sources of n-3 FA in both regions.

In conclusion, in this study the food consumption patterns for n-3 FA differ by location. N-3 FA intakes of elderly men living in the coastal region of Saudi Arabia were higher than those of men living in internal region of the country. Seafood consumption may be one of the reasons for higher intakes of $n-3 F A$ in the coastal region. The food contributing the most $n-3$ FA intakes in both coastal and internal residents was English walnuts. Some plant sources such as English walnuts may be considered a good source of n-3 FAs .

Future studies in Saudi Arabia may include both men and women of various age. Since not much has been reported on the effect of geographic location on the consumption of n-3 FAs in the United States and other countries, it is important to investigate this effect. Nutrition education intervention among internal residents is needed for increasing the consumption of n-3 FAs.

\section{References}

Bang, H.O., J. Dyerberg and H.M. Sinclair, 1980. The composition of the Eskimo food in north western Greenland. Am. J. Clin. Nutr., 33: 2657-2661.

Beaton, G.H., J. Burema and C. Ritenbangh, 1997. Errors in the interpretation of dietary assessments . Am. J. Clin. Nutr., 65: 1100s-1107s.

Bulliyya, G., K.K. Reddy, G.P.R. Reddy, P.C. Reddy, P. Reddanna and K.S. Kumari, 1990. Lipid profile among fish consuming coastal and non-fish consuming inland population. Eur. J. Clin. Nutr., 44: 481-485.

Charnock, J.S., 1999. Omega-3 polyunsaturated fatty acids and ventricular fibrillation: the possible involvement of eicosanoids. Prostaglandins Leukotrienes Essent. Fatty Acids, 61: 243-247.

Daviglus, M.L., J. Stamler, A.J. Orencia, A.R. Dyer, K. Liu, P. Greenland, M.K. Walash, D. Morries and R.B. Shekelle, 1997. Fish consumption and the 30-year risk of fatal myocardial infarction. N. Engl. J. Med., 336: 1046-1053.

Dietary Reference Intakes for Energy, Carbohydrates, Fiber, Fat, Protein and Amino Acids (Macronutrients), 2002. Food and Nutrition Board (FNB), Institute of Medicine, of the National. Academies, Available at:<http://www.books. nap.edu/books/03098573/html/RI.html>. Accessed, April 9, 2002.

Dolecek, T.A., 1992. Epidemiological evidence of relationships between dietary polyunsaturated fatty acids and mortality in the multiple risk factors intervention trial: dietary PUFA and mortality. Proc. Soc. Exp. Biol. Med., 200: 177-182. 


\section{AL-Numair et al.: Omega-3 Fatty Acid Consumption and Food Sources}

Dyerberg, J., H.O. Bang, E. Stoffersen, S. Monacada and J.R. Vane, 1978. Eicosapentaenoic acid and prevention of thrombosis and arteriosclerosis. Lanect, 2: 117-119.

Duo, L.I and A.J. Sinclair, 2001 The alpha linolenic acid content of green vegetales commonly available on Aunstralia. Int. J. Vitam. Nutr. Res., 71: 223-228.

Food Processor Plus computer program software. (ESHA Version 0.7; Salem, Oregon, 2002).

Gerster, H., 1998. Can adults adequately convert alpha linolinc acid (18:3n-3) to eicosapentaenoic acid (20:5n-3), and docosahexaenoic acid (22:6n-3). Int. J. Vitam. Nutr. Res., 68: 159-173.

Gil, A., 2002. Polyunsaturated fatty acids and inflammatory diseases. Biomed. Pharmacother., 56: 388-396.

GISSI-Prevention investigators, 1999. Dietary supplementation with omega-3 poly- unsaturated fatty acids and vitamin $\mathrm{E}$ after myocardial infarction. Results of the GISSI - prevention Trial. Lancet, 354: 447-455.

Harper, C.R. and T. A. Jacobson, 2001. The Fats of Life. Arch. Inter. Med.,161: 2185-2192.

Harris, W.S., 1996. N-3 fatty acids and lipoproteins: comparison results from human and animal studies. Lipids, 31: 243-252.

Holub, B.J., 2002. Clinical Nutrition: 4. Omega-3 fatty acids in cardiovascular care. Can. Med. Assoc. J., 166: 608-615.

Horrocks, L.A. and Y.Y. Yeo, 1999. Health benefits of docosahexaenoic acid. Pharmacol. Res., 40: 211225.

Howe, PR., 1997. Dietary fats and hypertension: focus on fish oil. Ann. N. Y. Acad. Sci., 827: 339-352.

Hu, F.B., M.J. Stampfer, J.E. Manson, E.B. Rimm, G.A. Colditz, B.A. Rosner, F.E. Speizer, C.H. Hennekens and W.C. Willett, 1998. Frequent nut consumption and risk of coronary heart disease in among women: prospective cohort study. Br. Med. J., 317: 1341-1345.

Hu, F.B., M.J. Stampfer, J.E. Manson, E.B. Rimm, A. Wolk, G.A. Colditz, C.H. Hennekens and W.C. Willet, 1999. Dietary intake of "-linolenic acid and risk of fatal ischemic heart disease women. Am. J. Clin. Nutr., 69: 890-895.

Hu, F.B., W. Willett, M.J. Stamperfer, K.M. Rexrode, C.M. Albert, D. Hunter and J.E. Manson, 2002. Fish and omega - 3 fatty acid intake and risk of coronary heart disease in women. JAMA., 787: 1815-1821.

James, M.J., R.A. Gibson and L.G. Cleland, 2000. Dietary polyunsaturated fatty acids and inflammatory mediator producation. Am. J. Clin. Nutr., 71: 343s348s.
Kotab, A.R., A.F. Hadeed, and A.A. Abdulla, 1991. Omega-3 polyunsaturated fatty acid content of some popular species of Arabian Gulf fish. Food Chem., 40: 185-190.

Kris-Etherton, P.M., W.S. Harris and L.J. Appel, 2002. AHA Scientific statement .Fish consumption, fish oil, omega-3 fatty acids, and cardiovascular disease. Circulation., 106: 2747-2757.

Lemaitre, R.N., I.B. King, D. Mozaffarian, L.H. Kuller, R.P. Tracy and D.S. Siscovick, 2003. n-3 polyunsaturated fatty acids, fatal ischemic heart disease, and nonfatal myocardial infarction in older adults: the Cardiovascular Health Study. Am. J. Clin. Nutr., 77: 319-325.

Lewis, N.M., K. Schlach and S.E. Scheideler, 2000. Serum lipid responses to $n-3$ fatty acid enriched eggs in persons with hypercholesterolemia. J. Am. Diet. Assoc., 100: 365-367.

Lewis, N.M., A.C. Widga, J.S. Buck and A.M. Frederick, 1995. Survey of omega-3 fatty acids in diets of Midwest low-income pregnant women. J. Agromed., 2: 49-57.

Mori, T.A., D.Q. Bao, V. Burke, I.B. Puddely and L.J. Beillin, 1999. Docosahexaenoic acid but not eicosapentaenoic acid lowers ambulatory blood pressure and heart rate in humans. Hypertension, 34: 253-260.

Nettleton, J.A., 1991. N-3 fatty acids: comparison of plant and seafood source in human nutrition. J. Am. Diet. Assoc., 91: 331-337.

Prineas, R.J., L.H. Kushi and A.R. Folsom, 1993. English English walnuts and serum lipids. N. Engl. J. Med., 328: 603-607.

Saudi Arabian Information Resource; Saudi Ministry of Information, 2002. Available at: http:// www.Saudinf.com/ main. Accessed, April 10, 2002.

Simopoulos, A.P., 2002. Omega-3 fatty acids in inflammation and autoimmune diseases. J. Am. Coll. Nutr., 21: 495-505.

Statistical Package for the Social Sciences computer program software. (SPSS for windows, version 11.0, SPSS. Inc, 2002.

Statistical Yearbook, 1999. Kingdom of Saudi Arabia. Ministry of Planning. Central Department of Statistics.

Torres, I.C., L. Mira, C.P. Ornelas and A. Melim, 2000. Study of the effect of dietary fish intake on serum lipids and lipoprotein in two populations with different dietary habits. Br. J. Nutr., 83: 371-379.

United States Department of Agricultures' Food Guide Pyramid, 2000. Available at: http:// www.nal.usda. gov: 8001/py/pmap.htm. Accessed, September 8, 2003. 